\title{
Aberrant Interhemispheric Connectivity in Obstructive Sleep Apnea-Hypopnea Syndrome
}

\author{
Yu-Ting Liu', Hui-Xin Zhang ${ }^{2}$, Hui-Jun Li', Ting Chen², Ya-Qing Huang', Lian Zhang ${ }^{3}$, \\ Zhi-Chun Huang ${ }^{3}$, Bin Liu ${ }^{4}$ and Ming Yang ${ }^{1 *}$
}

${ }^{1}$ Department of Radiology, Children's Hospital of Nanjing Medical University, Nanjing, China, ${ }^{2}$ School of Medicine, Southeast University, Nanjing, China, ${ }^{3}$ Department of Otolaryngology-Head and Neck Surgery, Zhongda Hospital, Southeast University, Nanjing, China, ${ }^{4}$ Department of Radiology, Zhongda Hospital, Southeast University, Nanjing, China

OPEN ACCESS

Edited by:

Ahmed S. BaHammam, King Saud University,

Saudi Arabia

Reviewed by:

Masoud Tahmasian,

Shahid Beheshti University, Iran

Michael K. Scullin,

Baylor University, United States

${ }^{*}$ Correspondence:

Ming Yang

yangming19710217@163.com

Specialty section:

This article was submitted to

Sleep and Chronobiology,

a section of the journal

Frontiers in Neurology

Received: 07 January 2018

Accepted: 20 April 2018

Published: 08 May 2018

Citation:

Liu Y-T, Zhang H-X, Li H-J, Chen T, Huang $Y-Q$, Zhang L, Huang Z-C, Liu B and Yang M (2018) Aberrant Interhemispheric Connectivity in Obstructive Sleep ApneaHypopnea Syndrome.

Front. Neurol. 9:314.

doi: 10.3389/fneur.2018.00314
Objective: To determine the changes in interhemispheric functional coordination in patients with obstructive sleep apnea-hypopnea syndrome (OSAHS) relative to controls, using a recently introduced method of analysis: voxel-mirrored homotopic connectivity (VMHC).

Methods: Twenty-nine patients with OSAHS and twenty-six normal sex-, age-, and education-matched controls were recruited and resting-state functional magnetic resonance imaging data were obtained. We employed VMHC to analyze the interhemispheric functional connectivity differences between groups. The $z$-values of alterations in VMHC in brain region were correlated with clinical characteristics.

Results: Compared with controls, patients with OSAHS had significantly higher scores for body mass index ( $t=5.749, P<0.001)$, apnea-hypopnea index $(\mathrm{AHI} ; t=7.706$, $P<0.001)$, oxygen desaturation index $(t=6.041, P<0.001)$, and Epworth sleepiness scale ( $t=3.711, P<0.001$ ), but significantly lower scores on the Rey-Osterrieth complex figure test-immediate recall $(t=-3.727, P<0.05)$. On the same basis, the VMHC showed significant increases in bilateral calcarine cortex and precuneus. Moreover, significant, positive correlations were found in only these areas between the $\mathrm{AHI}$ and the VMHC change coefficients $(r=0.399, P=0.032 ; r=0.378, P=0.043)$.

Conclusion: We found a memory defect in patients with OSAHS. The correlation between the abnormal VMHC and the AHI in patients with OSAHS suggested that $\mathrm{AHI}$ might be a key factor in cognitive dysfunction, which might offer new insights into the neural pathophysiology underlying OSAHS-related cognitive deficits.

Keywords: resting-state functional magnetic resonance imaging, obstructive sleep apnea-hypopnea syndrome, voxel-mirrored homotopic connectivity, functional connectivity, cognitive deficits

\section{INTRODUCTION}

Obstructive sleep apnea-hypopnea syndrome (OSAHS) is a common, chronic sleep disease, whose characteristics include repeated episodes of partial or complete obstruction of the upper airway, and continual diaphragmatic efforts to breathe during sleep (1). Demographic studies show that the incidence of obstructive sleep apnea for middle-aged people is $2-4 \%$, making it a major public

Abbreviations: AHI, apnea-hypopnea index; CFT, Rey-Osterrieth complex figure test; DMN, default mode network; ESS, Epworth sleepiness scale; FC, functional connectivity; LMT, logical memory test; MMSE, mini-mental state examination; MRI, magnetic resonance imaging; ODI, oxygen desaturation index; OSAHS, obstructive sleep apnea-hypopnea syndrome; VMHC, voxel-mirrored homotopic connectivity. 
health problem (2). Several cognitive domains could be affected by OSAHS, resulting in psychomotor dysfunction, memory weakness, decrements in vigilance and attention, and executive dysfunctions (3-5). Over the last 20 years, it has been shown that OSAHS can produce functional and structural alterations in the brain (6-8). Several studies using a voxel-based morphometry method found gray matter loss in patients with OSAHS compared with healthy controls in multiple brain regions, including posterior lateral parietal cortex, inferior temporal gyrus, hippocampus, parahippocampal gyrus, anterior cingulate gyrus, quadrangular lobule, and cerebellum (9-11). Using diffusion tensor imaging-based mean diffusivity procedures, global brain mean diffusivity values were found to be significantly decreased in patients with OSAHS. Some brain regions are especially affected, which may be a result of axonal, glial, and other cell alterations (12). In arterial spin labeling imaging, patients with OSAHS showed decreased cerebral blood flow values in multiple bilateral brain regions, including superior cerebellar peduncle, corticospinal tract, and pontocerebellar tract (13). Magnetic resonance spectroscopy has proved to be a useful neuroimaging tool able to detect alterations in cerebral metabolism, which may reflect pathological insults to brain integrity. Several magnetic resonance spectroscopy studies have revealed remarkable metabolic alterations in OSAHS (14-16).

Resting-state functional magnetic resonance imaging (MRI) based on blood-oxygenation-level dependent contrast (perhaps better known as BOLD contrast) has been widely used in various functional connectivity (FC) studies ranging from examinations of psychiatric disorders to neurological conditions (17), as well as human brain function studies (18-20). Previous neuroimaging studies have demonstrated altered brain function and connectivity in patients with OSAHS. The main findings are as follows: (1) significantly reduced FC within the anterior default mode network (DMN), bilateral fronto-parietal network, and sensorimotor network but increased FC within the posterior DMN (21), (2) the FC within the DMN, especially between the insula and the other DMN regions, is disrupted, and this abnormal FC associates with the severity of OSAHS $(22,23)$, and (3) resting-state FC disturbances of DMN are present in patients with OSAHS, as revealed by CONN software in our previous study (24).

As the largest commissural fiber bundle, the corpus callosum can facilitate communication and integration of emotional, cognitive, motor, and sensory information between the two cerebral hemispheres $(25,26)$. Despite its importance, little information is available regarding alterations in interhemispheric FC in patients with OSAHS. Diffusion tensor imaging has demonstrated changes in white matter integrity in the corpus callosum of these patients (27). Pathological alterations of the corpus callosum could impair the interhemispheric functional interactions that are foundational for the integration of executive control and attentional processing $(28,29)$. This is consistent with the observation of dysfunctions of executive control and attention in patients with OSAHS (3-5). In general, these studies raise the question of whether there are significant differences in interhemispheric functional coordination between patients with OSAHS and normal controls.

To explore this issue, we used calculations of voxel-mirrored homotopic connectivity (VMHC) to investigate interhemispheric FC. This method is a voxel-wise measurement of functional homotopy that reveals the synchrony of resting-state BOLD fluctuations between a voxel in one hemisphere and its mirror-image counterpart in the other. VMHC has been successfully applied to investigate interhemispheric functional homotopy in several diseases including autism, cocaine addiction, and schizophrenia (30-34). VMHC is an emerging method of analyzing the restingstate, functional magnetic resonance image (rs-fMRI). As far as we know, ours is the first study to use it to explore interhemispheric synchrony and thus to obtain correlations between measures of interhemispheric FC and clinical variables in OSAHS.

\section{MATERIALS AND METHODS}

\section{Subjects}

Twenty-nine newly diagnosed, untreated patients with OSAHS and twenty-six normal gender-, education-, and age-comparable controls were recruited by the Sleep Laboratory of the Otolaryngology-Head and Neck Surgery Department of Zhongda Hospital, Southeast University. No patient had comorbid conditions such as heart failure or central nervous system diseases (e.g., epilepsy, stroke, and tumor) or a history of psychotropic drug use. Patients with a history of airway, laryngeal, or pharyngeal surgery were also excluded. All controls were healthy, without any history of brain abnormalities or heart failure. All participants in both the OSAHS and control groups were between 20 and 60 years of age, right-handed, and free of metallic implants or other contraindications for MRI scan.

This study was carried out in accordance with the recommendations of the Institutional Ethics Committee of Zhongda Hospital, Southeast University, Nanjing. Before the study, all participants gave written, informed consent in accordance with the Declaration of Helsinki and the protocol was approved by the Institutional Ethics Committee of Zhongda Hospital, Southeast University, Nanjing.

\section{Cognition and Sleep Assessment}

We used the mini-mental state examination (MMSE) to screen for dementia and cognitive impairments (35). The Rey-Osterrieth complex figure test (CFT) and the logical memory test (LMT) of the Wechsler memory scale-revised were used for evaluating memory, attention, and executive functions (36). The Epworth sleepiness scale (ESS) was applied to evaluate sleep quality and daytime sleepiness (37).

\section{Polysomnography}

The night before functional MRI (fMRI) scanning, the OSAHS and control groups were asked to undergo polysomnography monitoring (YH-2000A system) in the Department of Otolaryngology-Head and Neck Surgery of Zhongda hospital. All participants were prohibited from drinking alcohol or caffeinated beverages for $12 \mathrm{~h}$ before polysomnography. For all participants, polysomnography was performed from 11:00 p.m. to 07:00 a.m. A standard encephalogram (nine channels, used to distinguish between sleep and wakefulness, sleep stages, and to measure the proportion of sleep stages), chin electromyography, electrocardiography, oculography, oral cavity and nasal cavity 
airflow, movements of the chest and abdomen, oximeter, physical position, and stertor were recorded. Two indexes related to sleep were then calculated in accordance with the criteria of the American Academy of Sleep Medicine $(38,39)$. These were apnea-hypopnea index (AHI, total number of hypopnea and apnea events per hour of sleep) and oxygen desaturation index (ODI, the number of desaturation events per hour of sleep). A hypopnea was defined as a drop in breathing extent of $30 \%$ lasting at least $10 \mathrm{~s}$, associated with repeated respiratory effort and a drop in oxygen saturation of at least $4 \%$. An obstructive apnea was defined as a drop in respiratory amplitude of $90 \%$ lasting at least $10 \mathrm{~s}$, associated with continued or increased inspiratory effort. A desaturation was defined as a drop in oxygen saturation of at least four percent lasting at least $3 \mathrm{~s}$.

\section{Magnetic Resonance Imaging}

Functional and structural MRI scans were performed on all participants at the Radiology Department of Zhongda Hospital using a 3.0 T MRI scanner (MAGENETOMTrio, Siemens, Erlangen, Germany). We adopted earplugs to reduce scanning noise and foam padding to decrease head motion. All participants were asked to close their eyes and not sleep, not to think about anything in particular, and to avoid any head motion as far as possible during scanning. The rs-fMRI data were acquired axially by applying an echo-planar imaging sequence sensitive to BOLD contrast. One hundred eighty functional images were obtained covering the whole brain (repetition time 2,000 ms, echo time $25 \mathrm{~ms}$, flip angle $90^{\circ}$, field of view $240 \mathrm{~mm} \times 240 \mathrm{~mm}$, matrix $64 \times 64$, slice number 36, slice thickness $4 \mathrm{~mm}$, no gap, aggregate duration of scan 6 min and $6 \mathrm{~s}$ ). High-spatial-resolution, T1-weighted images were also obtained from each participant by applying a magnetic prepared gradient echo sequence, yielding 176 structural images (repetition time $1,900 \mathrm{~ms}$, echo time $2.48 \mathrm{~ms}$, flip angle $9^{\circ}$, field of view $256 \mathrm{~mm} \times 256 \mathrm{~mm}$, matrix $256 \times 256$, slice thickness $1 \mathrm{~mm}$, no gap, duration $4 \mathrm{~min}$ and $18 \mathrm{~s}$ ). Furthermore, 20 fluid-attenuated inversion-recovery images (perhaps better known as FLAIR images) were acquired to screen for structural brain lesions (repetition time 8,500 ms, echo time $94 \mathrm{~ms}$, slice thickness $5 \mathrm{~mm}$, duration $1 \mathrm{~min}$ and $59 \mathrm{~s}$ ). The total duration of scanning was $12 \mathrm{~min}, 23 \mathrm{~s}$.

\section{MRI Data Processing}

Functional MRI data analysis was performed using DPARSFA (rs-fMRI Advanced Edition) with statistical parametric mapping $\left(\mathrm{SPM} 8^{1}\right)$ and rs-fMRI data analysis toolkits $\left(\mathrm{REST}^{2}\right)$ with the MATLAB 2010a platform (40). A total of 180 volumes were acquired. The first 10 volumes were discarded because of instability in the initial MRI signals and adaptation of the participants to the scanner environment, and the remaining 170 volumes were submitted to the following processing, beginning with slice timing adjustment for acquisition time delays between different slices and realignment for head motion correction. (Any participant whose head motions were more than $2.0-\mathrm{mm}$ of translation or more than $2.0^{\circ}$ of rotation was excluded.) Then the fMRI data

${ }^{1}$ http://www.fil.ion.ucl.ac.uk/spm/ (Accessed: 2010).

${ }^{2}$ http://www.restfmri.net/ (Accessed: 2011). were spatially normalized to the Montreal Neurological Institute template with resampling to a $3 \mathrm{~mm} \times 3 \mathrm{~mm} \times 3 \mathrm{~mm}$ voxel size, smoothing with an isotropic Gaussian kernel (full-width at half maximum $=4 \mathrm{~mm}$ ) and finally, detrending and bandpass filtering at $0.01-0.08 \mathrm{~Hz}$.

\section{VMHC Analysis}

We used REST software to compute the VMHC. The Pearson's correlation coefficient between the remaining time series of each voxel and that of its mirrored interhemispheric voxel was calculated and the homotopic FC was measured (41). Subsequently, the correlation values were converted to $z$-values using Fisher's $r$-to- $z$ transformation to enhance the stability of the values. The resulting values were considered a measure of VMHC and were used for the final intergroup analysis.

\section{Statistical Analysis}

A two-sample $t$-test was used for VMHC-map analysis to investigate altered interhemispheric FC in patients with OSAHS. $P<0.05$ was considered statistically significant, and cluster sizes were set at 63 voxels, as determined by Monte Carlo simulations using the AFNI AlphaSim program. Because of the influence of micromovements from volume to volume on the FC (42), framewise displacement values were calculated to reflect the temporal derivative of the movement parameters for each patient. Analysis revealed no remarkable difference in the mean framewise displacement values between patients with OSAHS and normal controls. Finally, a Pearson correlation analysis was performed to explore the correlations between VMHC and clinical variables relevant to OSAHS including AHI, ODI, body mass index (BMI), MMSE, CFT, LMT, and ESS. Partial correlation with some covariates (age, sex, and years of education) was performed to evaluate the effects of these covariates on increased VMHC.

Two-sample $t$-tests and $\chi^{2}$-tests were used to analyze differences in demographic data between patients with OSAHS and normal controls. $P<0.05$ was considered to indicate statistical significance.

\section{RESULTS}

\section{Demographic and Clinical Data}

In our study, 55 participants (29 patients with OSAHS and 26 normal controls) were included in the final data analysis. The demographic and clinical data of all participants are presented in Table 1. No significant differences were found between patients with OSAHS and controls in age, sex, or years of education $(P>0.05)$. However, the patients with OSAHS had significantly higher scores for BMI $(t=5.749, P<0.001)$, AHI $(t=7.706$, $P<0.001)$, ODI $(t=6.041, P<0.001)$, and ESS $(t=3.711$, $P<0.001)$, but significantly lower scores for CFT-immediate recall $(t=-3.727, P<0.05)$ compared with controls. No significant differences were observed between patients with OSAHS and controls in CFT-delayed recall $(t=-1.862, P>0.05)$, LMT-immediate recall $(t=-0.322, P>0.05)$, LMT-delay recall $(t=-0.371, P>0.05)$, or score on the MMSE $(t=-1.439$, $P>0.05)$. 


\section{Changes in VMHC in Patients With OSAHS Compared With Normal Controls}

Patients with OSAHS showed significantly increased VMHCs in the calcarine cortex and precuneus compared with healthy controls. However, relatively decreased VMHCs were not seen

TABLE 1 | Population statistics and clinical information.

\begin{tabular}{lcccrr}
\hline Characteristics & Controls (26) & OSAHS (29) & $\boldsymbol{P}$ value & $\boldsymbol{t}$ Value & ES \\
\hline Age & $34.46 \pm 9.97$ & $39.62 \pm 9.95$ & 0.061 & 1.918 & 0.250 \\
Sex (female/male) & $8 / 18$ & $6 / 23$ & 0.392 & 0.734 & 0.592 \\
Education (years) & $13.96 \pm 2.58$ & $12.67 \pm 3.17$ & 0.103 & -1.658 & -0.218 \\
BMI & $22.16 \pm 2.93$ & $27.99 \pm 4.37$ & 0.000 & 5.749 & 0.617 \\
AHI & $2.43 \pm 1.68$ & $33.67 \pm 21.75$ & 0.000 & 7.706 & 0.711 \\
ODI & $0.93 \pm 1.03$ & $32.07 \pm 27.73$ & 0.000 & 6.041 & 0.621 \\
MMSE & $28.96 \pm 1.51$ & $28.34 \pm 1.65$ & 0.156 & -1.439 & -0.192 \\
CFT-immediate & $35.92 \pm 0.39$ & $35.17 \pm 1.00$ & 0.001 & -3.727 & -0.442 \\
recall & & & & & \\
CFT-delay recall & $21.88 \pm 5.96$ & $18.48 \pm 7.41$ & 0.068 & -1.862 & -0.245 \\
LMT-immediate & $20.69 \pm 6.19$ & $20.07 \pm 7.93$ & 0.749 & -0.322 & -0.043 \\
recall & & & & & \\
LMT-delay recall & $19.77 \pm 5.88$ & $19.03 \pm 8.41$ & 0.712 & -0.371 & -0.051 \\
ESS & $4.81 \pm 2.61$ & $7.69 \pm 3.09$ & 0.000 & 3.711 & 0.449 \\
& & & & &
\end{tabular}

Averages are accompanied by the 95\% confidence limits.

OSAHS, obstructive sleep apnea-hypopnea syndrome; ES, effect size; BMI, body mass index; AHI, apnea-hypopnea index; ODI, oxygen desaturation index; MMSE, mini-mental state examination; CFT, Rey-Osterrieth complex figure test; LMT, logical memory test of the Wechsler memory scale-revised; ESS, Epworth sleepiness scale. For the sex ratio, the $\chi^{2}$ value substitutes for the $t$-value. in patients with OSAHS. The details are presented in Figures 1 and $\mathbf{2}$ and Table 2.

\section{Correlation Analysis}

In patients with OSAHS, a significant positive correlation was found in the precuneus between VMHC values and AHI $(r=0.378$, $P=0.043$ ). In addition, a significant positive correlation was found in the calcarine cortex between VMHC values and AHI $(r=0.399$, $P=0.032$ ) (see Figures 3 and 4). VMHC did not associate with ODI, BMI, MMSE, CFT, or ESS. There were no significant correlations between increased VMHC and any covariate (age, $P=0.676$, $r=0.106$; gender, $P=0.433, r=0.197$; years of education, $P=0.636$, $r=-0.120)$.

\section{DISCUSSION}

In this study, we adopt a VMHC approach to investigate possible differences in interhemispheric FC between patients with OSAHS and controls. We found a significant increased VMHC in the precuneus and calcarine cortex in patients with OSAHS compared with controls. What is more, there were significant positive correlations between the AHI and the VMHC in these brain areas.

Few past studies have reported increased activities in the precuneus in patients with OSAHS. However, here we found significantly increased VMHC in the precuneus of such patients. Huynh and coworkers found no difference in gray matter volume

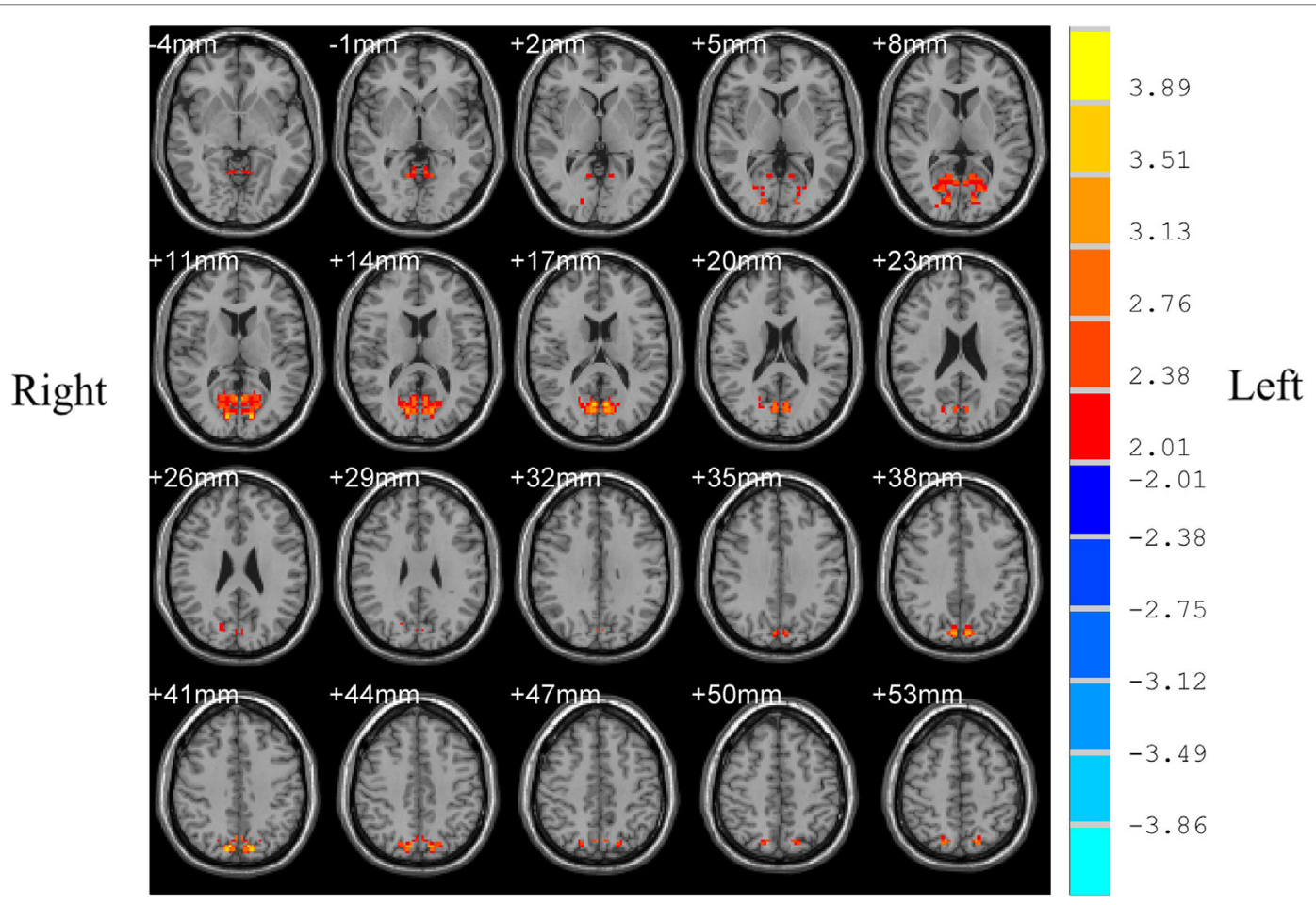

FIGURE 1 | Shown is a significantly increased voxel-mirrored homotopic connectivity (VMHC) in obstructive sleep apnea-hypopnea syndrome patients as compared with controls. Thresholds are set at a corrected $P<0.05$, as determined by Monte Carlo simulation. Red indicates increased VMHC. The left side of the images represents the right of the brain and vice versa. 


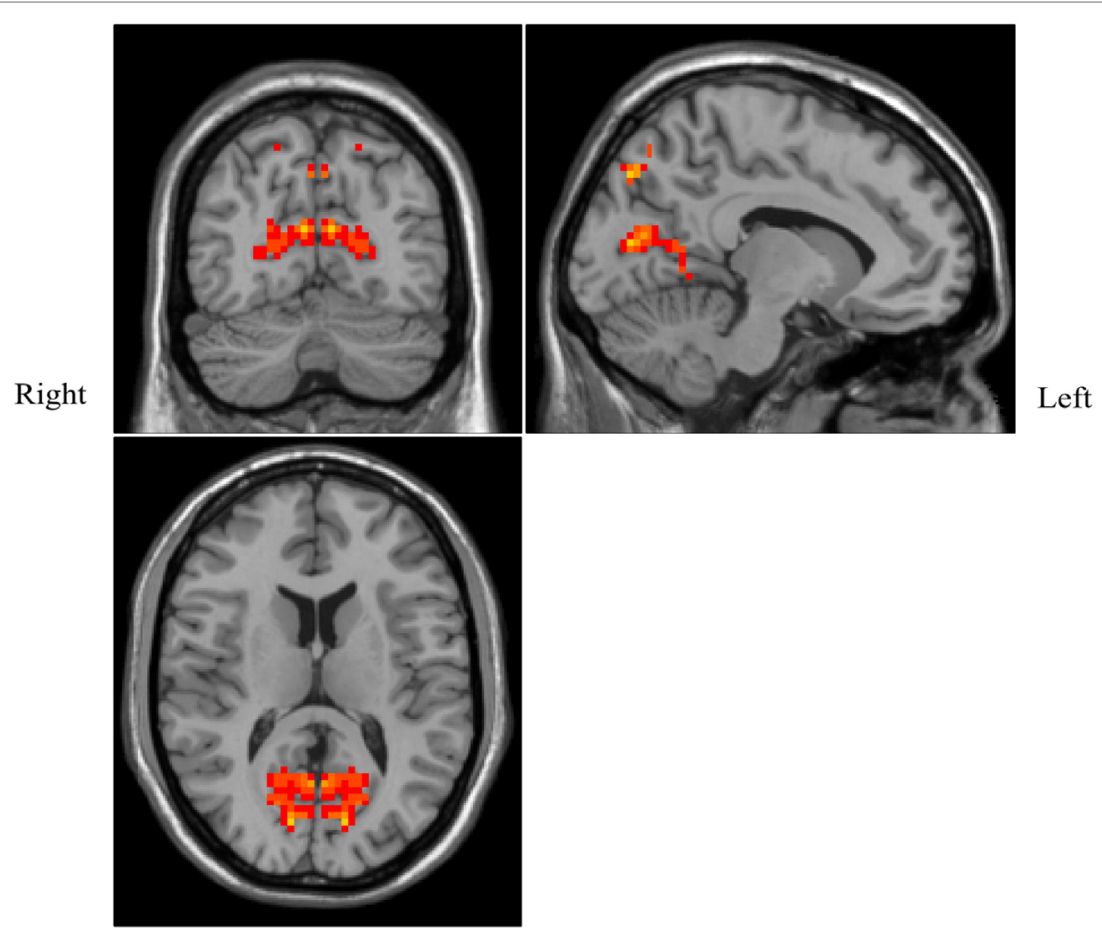

FIGURE 2 | Shown is a significantly increased voxel-mirrored homotopic connectivity (VMHC) in obstructive sleep apnea-hypopnea syndrome patients as compared with controls. Thresholds are set at a corrected $P<0.05$, as determined by Monte Carlo simulation. Red indicates increased VMHC. The left side of the images represents the right of the brain and vice versa.

TABLE 2 | Brain areas with differences in voxel-mirrored homotopic connectivity between OSAHS patients and controls.

\begin{tabular}{lrccr}
\hline Brain area & BA & MNI & $\boldsymbol{t}$ Value & Voxels \\
\hline Precuneus & 7 & $\pm 9,-78,42$ & 4.023 & 63 \\
Calcarine & 18 & $\pm 6,-69,18$ & 3.545 & 198 \\
\hline
\end{tabular}

BA, Brodmann area; MNI, Montreal Neurological Institute coordinates; OSAHS, obstructive sleep apnea-hypopnea syndrome.

between patients with moderate-to-severe OSAHS and normal controls, so an increased VMHC was not attributed to structural differences, but possibly to strengthened neural activity (43). Our findings are consistent with a previous report of an abnormal overactivation in the precuneus of OSAHS patients. That report suggested that the overactivation represents an adaptive compensatory response (44).

The precuneus is part of the posterior DMN, which plays an important role in fundamental cognitive functioning, including episodic memory retrieval, visuo-spatial imagery, self-processing, and consciousness $(45,46)$. The DMN involves a set of brain regions that are more active during rest than during goal-directed tasks and is involved in a wide range of higher-order cognitive functions (47). Several systematic meta-analytic studies have highlighted the importance of the DMN in cognition and clinical symptoms $(6,48)$. It has been reported that abnormal activity in the DMN, such as abnormal resting-state FC among its sub regions, significant regional deficits in spontaneous activity, and a decrease of blood-oxygenation-dependent fluctuations in its main nodes, correlate with sleep parameters and delay memory (49-51). These findings imply the presence of cognitive deficits in OSAHS patients. Similarly, the altered VMHC in the precuneus demonstrated here implies a dysfunction of the DMN, which might suggest the presence of cognitive impairments in the OSAHS patients of our study.

Apart from any cognitive deficits that can arise from these abnormalities, these changes imply problems in sustained and divided attention that arguably contribute to the well-recognized driving difficulties of patients with OSAHS (52) and may also lead to dysfunctional sleep rhythms (53). Moreover, we also found a positive correlation between enhanced VMHC and the AHI in patients with OSAHS. It has long been speculated that repeated hypopnea leads to aberrant properties in the DMN and precuneus, resulting from an adaptive compensatory response tending to maintain the normal activities of the brain.

The calcarine cortex is a main core of the visual recognition network (54), and has been associated with attentional shifts to an expected visual goal and with modulation of visual input by attention, especially when visual information is used to guide saccades or reaching (55). Previous neuroimaging studies have shown that patients with OSAHS have impairment in several cognitive domains, including executive control function, episodic memory, coordination of movement, and attention (3-5). However, only a few studies have reported visual dysfunction in patients with OSAHS. Giora and coworkers found that in a visual task, patients with OSAHS had a significantly longer reaction times than controls, indicating the presence in these 


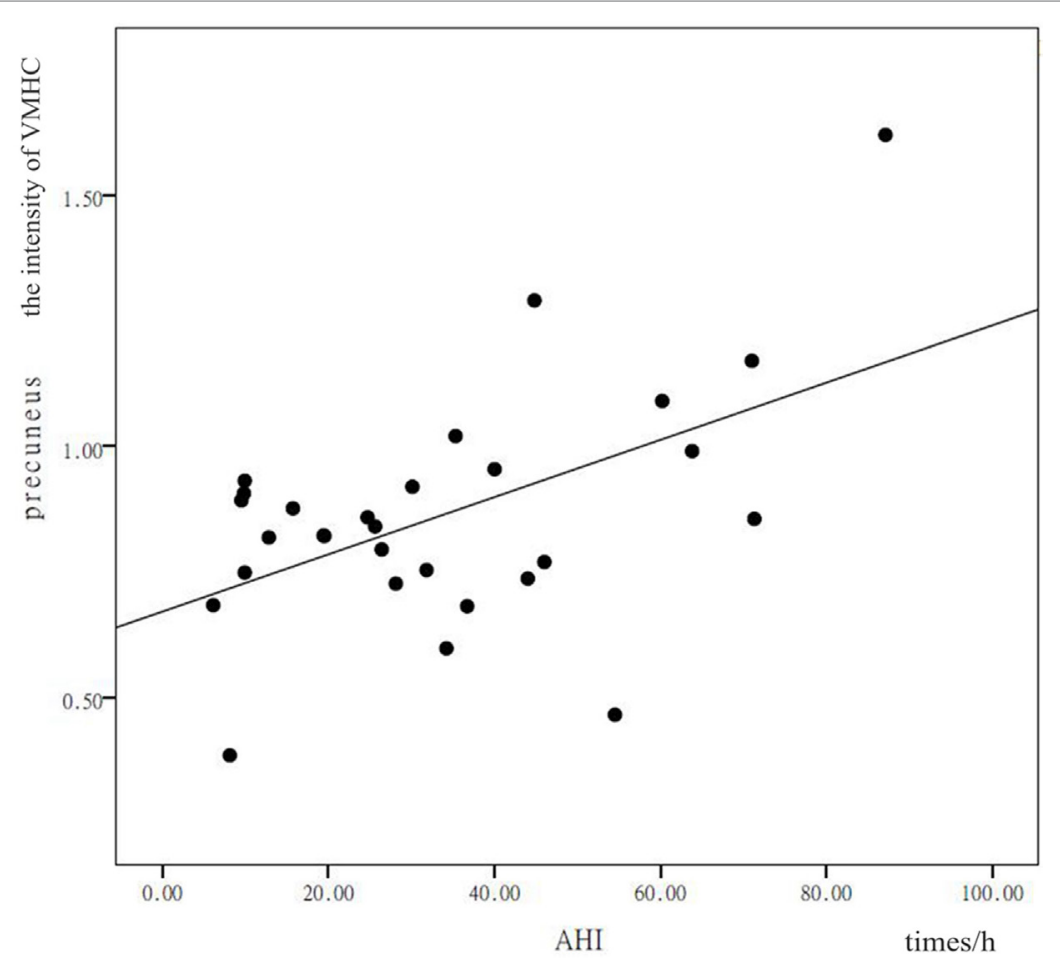

FIGURE 3 | Shown is a significant positive correlation between the voxel-mirrored homotopic connectivity (VMHC) values in the precuneus and calcarine cortex, and the apnea-hypopnea index (AHI). $X$ represents the $\mathrm{AHI}$ and $Y$ represents the intensity of the VMHC.

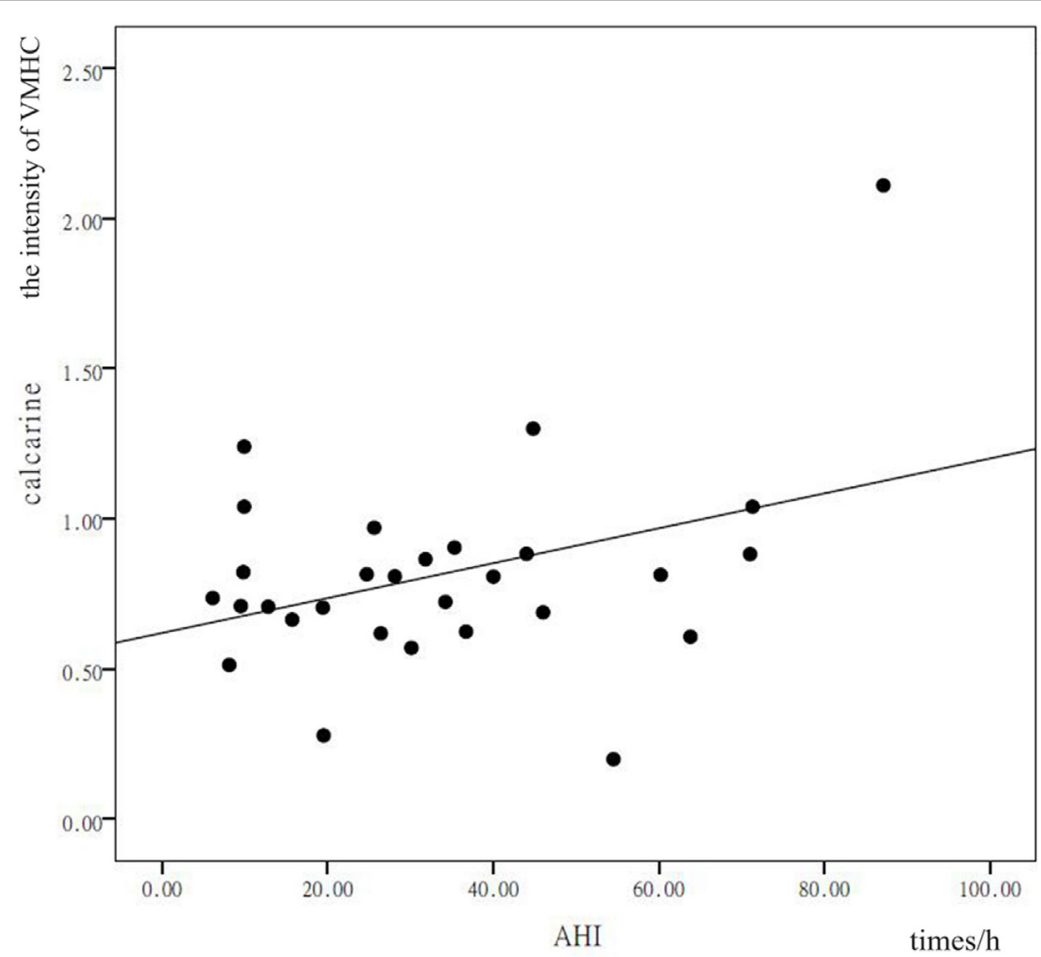

FIGURE 4 | Shown is a significant positive correlation between the voxel-mirrored homotopic connectivity (VMHC) values in the precuneus and calcarine cortex, and the apnea-hypopnea index $(\mathrm{AHI}) . X$ represents the $\mathrm{AHI}$ and $Y$ represents the intensity of the VMHC. 
patients of an impairment involving basic mechanisms of visual processing (56). Another study demonstrated that retinal nerve-fiber thickness is reduced in patients with OSAHS (57). It has also been reported that OSAHS leads to a high prevalence of open-angle glaucoma and visual-field defects $(58,59)$. In this study, the aberrant VMHC found in the calcarine cortex in the patients with OSAHS may indicate visual dysfunction. Furthermore, Chan and coworkers found impaired attention and visual-fine-motor coordination in children with OSAHS (60). Other research found an increased risk for traffic accidents in patients with OSAHS (61). We thus speculate that disorders of coordination of vision and motion might result in patients with OSAHS having an extra risk of positioning dislocations and accidents. In addition, since VMHC in the calcarine cortex here had a positive correlation with AHI, we suggest that AHI might be a key factor in visual dysfunction and that the calcarine area is sensitive to the effects of apnea and hypopnea.

Recently, a systematic meta-analysis of patients with OSAHS demonstrated co-activations in several bilateral brain regions including amygdala, hippocampus, thalamus, precuneus, and posterior cingulate cortex. This suggested a significant association of the disorder with affective and emotional processing, as well as with memory-related processes (62). Similarly, our results are largely in agreement with the above-mentioned findings, and it also follows that the dysfunctions of memory, attention, and executive control reported in patients with OSAHS might be partly explained by increased interhemispheric functional interactions in precuneus and calcarine cortex. Moreover, patients with OSAHS have an increased prevalence of several psychiatric disorders including major depressive disorder and anxiety (63). Studies have also found aberrant interhemispheric FC in patients with depression $(64,65)$. We speculate that malfunctions within interhemispheric FC could conceivably contribute to the genesis of the neuropsychiatric deficits previously reported to be prevalent in OSAHS, such as depression, emotional lability, and anxiety.

\section{CONCLUSION}

Interhemispheric functional interactions are at the foundation of the integration of executive control and attentional processing (61). Our study found increased homotopic connectivity

\section{REFERENCES}

1. Park JG, Ramar K, Olson EJ. Updates on definition, consequences, and management of obstructive sleep apnea. Mayo Clinic Proc (2011) 86:549-55. doi:10.4065/mcp.2010.0810

2. Young T, Palta M, Dempsey J, Skatrud J, Weber S, Badr S. The occurrence of sleep-disordered breathing among middle-aged adults. N Engl J Med (1993) 328:1230-5. doi:10.1056/NEJM199304293281704

3. Kim HC, Young T, Matthews CG, Weber SM, Woodward AR, Palta M. Sleep-disordered breathing and neuropsychological deficits: a populationbased study. Am J Respir Crit Care Med (1997) 156:1813-9. doi:10.1164/ ajrccm.156.6.9610026

4. Ferini-Strambi L, Baietto C, Di Gioia MR, Castaldi P, Castronovo C, Zucconi M, et al. Cognitive dysfunction in patients with obstructive sleep apnea (OSA):
(VMHC) in bilateral precuneus and calcarine cortex in patients with OSAHS. Moreover, we found positive correlations between enhanced VMHC in precuneus and calcarine cortex and the AHI. These results indicate that abnormal VMHC may provide an early biomarker for the detection of cognitive impairments in patients with OSAHS, advantageously using a noninvasive imaging technology. However, several limitations of this study should be noted. First, the study should be repeated with a larger sample size to eliminate any possible instability in the results. Second, some participants were unable to accommodate an encephalogram examination, so the encephalogram data in our study is incomplete. In the future, we will endeavor to obtain complete encephalogram data and examine the rapid-eye-movementspecific features of OSAHS. Third, we focused here on cognitive changes occurring in mixed-severity patients with OSAHS. A study specific to the mild-to-severe group should be conducted, which would offer powerful insights into neurophysiological mechanisms in patients with a distinct level of OSAHS. Finally, longitudinal studies are needed to provide imaging markers for clinical treatment.

\section{ETHICS STATEMENT}

All participants provided written informed consent before the study, and the protocol was approved by the institutional Ethics Committee of Zhongda Hospital, Southeast University, Nanjing.

\section{AUTHOR CONTRIBUTIONS}

$\mathrm{MY}, \mathrm{BL}$, and $\mathrm{Z}-\mathrm{CH}$ contributed conception and design of the study; Y-TL, H-XZ, TC, and LZ organized the database; Y-TL performed the statistical analysis; Y-TL wrote the first draft of the manuscript; H-JL and Y-QH wrote sections of the manuscript. All authors contributed to manuscript revision, read and approved the submitted version.

\section{FUNDING}

This study was supported by the Clinical Medical Science and Technology Special Project of Jiangsu, China (No. BL2013029), the Maternity and child care project, Jiangsu Province, China (F201554), and Science and technology development project, Nanjing, China (2015sc511023). partial reversibility after continuous positive airway pressure (CPAP). Brain Res Bull (2003) 61:87-92. doi:10.1016/S0361-9230(03)00068-6

5. Saunamaki T, Jehkonen M. A review of executive functions in obstructive sleep apnea syndrome. Acta Neurol Scand (2007) 115:1-11. doi:10.1111/j. 1600-0404.2006.00744.x

6. Khazaie H, Veronese M, Noori K, Emamian F, Zarei M, Ashkan K, et al. Functional reorganization in obstructive sleep apnoea (OSA) and insomnia: a systematic review of the resting-state fMRI. Neurosci Biobehav Rev (2017) 77:219-31. doi:10.1016/j.neubiorev.2017.03.013

7. Scullin MK. Do older adults need sleep? A review of neuroimaging, sleep, and aging studies. Curr Sleep Med Rep (2017) 3:204-14. doi:10.1007/ s40675-017-0086-Z

8. Weng HH, Tsai YH, Chen CF, Lin YC, Yang CT, Tsai YH, et al. Mapping gray matter reductions in obstructive sleep apnea: an activation 
likelihood estimation meta-analysis. Sleep (2014) 37:167-75. doi:10.5665/ sleep. 3330

9. Joo EY, Tae WS, Lee MJ, Kang JW, Park HS, Lee JY, et al. Reduced brain gray matter concentration in patients with obstructive sleep apnea syndrome. Sleep (2010) 33:235-41. doi:10.1093/sleep/33.2.235

10. Desseilles M, Dang-Vu T, Schabus M, Sterpenich V, Maquet P, Schwartz S. Neuroimaging insights into the pathophysiology of sleep disorders. Sleep (2008) 31:777-94. doi:10.1093/sleep/31.6.777

11. Morrell MJ, McRobbie DW, Quest RA, Cummin AR, Ghiassi R, Corfield DR. Changes in brain morphology associated with obstructive sleep apnea. Sleep Med (2003) 4:451-4. doi:10.1016/S1389-9457(03)00159-X

12. Kumar R, Chavez AS, Macey PM, Woo MA, Yan-Go FL, Harper RM. Altered global and regional brain mean diffusivity in patients with obstructive sleep apnea. J Neurosci Res (2012) 90:2043-52. doi:10.1002/jnr.23083

13. Yadav SK, Kumar R, Macey PM, Richardson HL, Wang DJ, Woo MA, et al. Regional cerebral blood flow alterations in obstructive sleep apnea. Neurosci Lett (2013) 555:159-64. doi:10.1016/j.neulet.2013.09.033

14. O'DonoghueFJ, WellardRM, RochfordPD,Dawson A,BarnesM, RuehlandWR, et al. Magnetic resonance spectroscopy and neurocognitive dysfunction in obstructive sleep apnea before and after CPAP treatment. Sleep (2012) 35:41-8. doi:10.5665/sleep.1582

15. Bartlett DJ, Rae C, Thompson CH, Byth K, Joffe DA, Enright T, et al. Hippocampal area metabolites relate to severity and cognitive function in obstructive sleep apnea. Sleep Med (2004) 5:593-6. doi:10.1016/j.sleep.2004. 08.004

16. Tonon C, Vetrugno R, Lodi R, Gallassi R, Provini F, Iotti S, et al. Proton magnetic resonance spectroscopy study of brain metabolism in obstructive sleep apnoea syndrome before and after continuous positive airway pressure treatment. Sleep (2007) 30:305-11. doi:10.1093/sleep/30.3.305

17. Guilleminault C, Poyares D, Rosa A, Huang YS. Heart rate variability, sympathetic and vagal balance and EEG arousals in upper airway resistance and mild obstructive sleep apnea syndromes. Sleep Med (2005) 6:451-7. doi:10.1016/j. sleep.2005.03.014

18. Sadaghiani S, Kleinschmidt A. Functional interactions between intrinsic brain activity and behavior. Neuroimage (2013) 80:379-86. doi:10.1016/j. neuroimage.2013.04.100

19. Laird AR, Eickhoff SB, Rottschy C, Bzdok D, Ray KL, Fox PT. Networks of task co-activations. Neuroimage (2013) 80:505-14. doi:10.1016/j. neuroimage.2013.04.073

20. Smith SM, Fox PT, Miller KL, Glahn DC, Fox PM, Mackay CE, et al. Correspondence of the brain's functional architecture during activation and rest. Proc Natl Acad Sci U S A (2009) 106:13040-5. doi:10.1073/pnas.0905267106

21. Zhang Q, Wang D, Qin W, Li Q, Chen B, Zhang Y, et al. Altered resting-state brain activity in obstructive sleep apnea. Sleep (2013) 36:651-9. doi:10.5665/ sleep. 2620

22. Park B, Palomares JA, Woo MA, Kang DW, Macey PM, Yan-Go FL, et al. Aberrant insular functional network integrity in patients with obstructive sleep apnea. Sleep (2016) 39:989-1000. doi:10.5665/sleep.5738

23. Zhang Q, Qin W, He X, Li Q, Chen B, Zhang Y, et al. Functional disconnection of the right anterior insula in obstructive sleep apnea. Sleep Med (2015) 16:1062-70. doi:10.1016/j.sleep.2015.04.018

24. Chen T, Yang M, Liu B, Liu YT, Zhang HX, Liu CC, et al. The resting-state functional connectivity of the default mode networks in patients with obstructive sleep apnea-hypopnea syndrome. CNS Neurol Disord Drug Targets (2017) 16:16-22. doi:10.2174/187152731601170111222000

25. Quigley M, Cordes D, Turski P, Moritz C, Haughton V, Seth R, et al. Role of the corpus callosum in functional connectivity. AJNR Am J Neuroradiol (2003) 24:208-12.

26. van der Knaap LJ, van der Ham IJ. How does the corpus callosum mediate interhemispheric transfer? A review. Behav Brain Res (2011) 223:211-21. doi:10.1016/j.bbr.2011.04.018

27. Kumar R, Pham TT, Macey PM, Woo MA, Yan-Go FL, Harper RM. Abnormal myelin and axonal integrity in recently diagnosed patients with obstructive sleep apnea. Sleep (2014) 37:723-32. doi:10.5665/sleep.3578

28. Gazzaniga MS. Cerebral specialization and interhemispheric communication: does the corpus callosum enable the human condition? Brain (2000) 123:1293-326. doi:10.1093/brain/123.7.1293

29. Hoptman MJ, Davidson RJ. How and why do the two cerebral hemispheres interact? Psychol Bull (1994) 116:195-219. doi:10.1037/0033-2909. 116.2 .195
30. Anderson JS, Druzgal TJ, Froehlich A, DuBray MB, Lange N, Alexander AL, et al. Decreased interhemispheric functional connectivity in autism. Cereb Cortex (2011) 21:1134-46. doi:10.1093/cercor/bhq190

31. Hoptman MJ, Zuo XN, D’Angelo D, Mauro CJ, Butler PD, Milham MP, et al. Decreased interhemispheric coordination in schizophrenia: a resting state fMRI study. Schizophr Res (2012) 141:1-7. doi:10.1016/j.schres.2012. 07.027

32. Kelly C, Zuo XN, Gotimer K, Cox CL, Lynch L, Brock D, et al. Reduced interhemispheric resting state functional connectivity in cocaine addiction. Biol Psychiatry (2011) 69:684-92. doi:10.1016/j.biopsych.2010.11.022

33. Chen HJ, Wang Y, Yang M, Zhu XQ, Teng GJ. Aberrant interhemispheric functional coordination in patients with HBV-related cirrhosis and minimal hepatic encephalopathy. Metab Brain Dis (2014) 29:617-23. doi:10.1007/ s11011-014-9505-8

34. Yuan K, Qin W, Liu P, Zhao L, Yu D, Zhao L, et al. Reduced fractional anisotropy of corpus callosum modulates interhemispheric resting state functional connectivity in migraine patients without aura. PLoS One (2012) 7:e45476. doi:10.1371/journal.pone.0045476

35. Shao C, Gu L, Mei Y, Li M. [Analysis of the risk factors of cognitive impairment in post-intensive care syndrome patient]. Zhonghua Wei Zhong Bing Ji Jiи Yi Хие (2017) 29:716-20. doi:10.3760/cma.j.issn.2095-4352.2017. 08.009

36. Shin MS, Park SY, Park SR, Seol SH, Kwon JS. Clinical and empirical applications of the Rey-Osterrieth complex figure test. Nat Protoc (2006) 1:892-9. doi:10.1038/nprot.2006.115

37. Johns MW. A new method for measuring daytime sleepiness: the Epworth sleepiness scale. Sleep (1991) 14:540-5. doi:10.1093/sleep/14.6.540

38. EEG arousals: scoring rules and examples: a preliminary report from the sleep disorders atlas task force of the American Sleep Disorders Association. Sleep (1992) 15:173-84. doi:10.1093/sleep/15.2.173

39. Redline S, Budhiraja R, Kapur V, Marcus CL, Mateika JH, Mehra R, et al. The scoring of respiratory events in sleep: reliability and validity. J Clin Sleep Med (2007) 3:169-200.

40. Chao-Gan Y, Yu-Feng Z. DPARSF: a MATLAB toolbox for "pipeline" data analysis of resting-state fMRI. Front Syst Neurosci (2010) 4:13. doi:10.3389/ fnsys.2010.00013

41. Zuo XN, Kelly C, Di Martino A, Mennes M, Margulies DS, Bangaru S, et al. Growing together and growing apart: regional and sex differences in the lifespan developmental trajectories of functional homotopy. J Neurosci (2010) 30:15034-43. doi:10.1523/JNEUROSCI.2612-10.2010

42. Power JD, Barnes KA, Snyder AZ, Schlaggar BL, Petersen SE. Spurious but systematic correlations in functional connectivity MRI networks arise from participant motion. Neuroimage (2012) 59:2142-54. doi:10.1016/j. neuroimage.2011.10.018

43. Huynh NT, Prilipko O, Kushida CA, Guilleminault C. Volumetric brain morphometry changes in patients with obstructive sleep apnea syndrome: effects of CPAP treatment and literature review. Front Neurol (2014) 5:58. doi:10.3389/ fneur.2014.00058

44. Castronovo V, Canessa N, Strambi LF, Aloia MS, Consonni M, Marelli S, et al. Brain activation changes before and after PAP treatment in obstructive sleep apnea. Sleep (2009) 32:1161-72. doi:10.1093/sleep/32.9.1161

45. Cavanna AE, Trimble MR. The precuneus: a review of its functional anatomy and behavioural correlates. Brain (2006) 129:564-83. doi:10.1093/brain/ awl004

46. Raichle ME, Macleod AM, Snyder AZ, Powers WJ, Gusnard DA, Shulman GL. A default mode of brain function. Proc Natl Acad Sci U S A (2001) 98:676-82. doi:10.1073/pnas.98.2.676

47. Buckner RL, Andrews-Hanna JR, Schacter DL. The brain's default network: anatomy, function, and relevance to disease. Ann N Y Acad Sci (2008) 1124:1-38. doi:10.1196/annals.1440.011

48. Shi Y, Chen L, Chen T, Li L, Dai J, Lui S, et al. A meta-analysis of voxel-based brain morphometry studies in obstructive sleep apnea. Sci Rep (2017) 7:10095. doi:10.1038/s41598-017-09319-6

49. Peng DC, Dai XJ, Gong HH, Li HJ, Nie X, Zhang W. Altered intrinsic regional brain activity in male patients with severe obstructive sleep apnea: a restingstate functional magnetic resonance imaging study. Neuropsychiatr Dis Treat (2014) 10:1819-26. doi:10.2147/NDT.S67805

50. Li HJ, Dai XJ, Gong HH, Nie X, Zhang W, Peng DC. Aberrant spontaneous low-frequency brain activity in male patients with severe obstructive sleep 
apnea revealed by resting-state functional MRI. Neuropsychiatr Dis Treat (2015) 11:207-14. doi:10.2147/NDT.S73730

51. Li HJ, Nie X, Gong HH, Zhang W, Nie S, Peng DC. Abnormal resting-state functional connectivity within the default mode network subregions in male patients with obstructive sleep apnea. Neuropsychiatr Dis Treat (2016) 12:203-12. doi:10.2147/NDT.S97449

52. Rosenzweig I, Weaver T, Morrell MJ. Obstructive sleep apnea and the central nervous system: neural adaptive processes, cognition and performance. 6th ed. In: Kryger MH, Roth T, editors. Principles and Practice of Sleep Medicine. Philadelphia, PA: Elsevier (2017).

53. Tagliazucchi E, Laufs H. Decoding wakefulness levels from typical fMRI resting-state data reveals reliable drifts between wakefulness and sleep. Neuron (2014) 82:695-708. doi:10.1016/j.neuron.2014.03.020

54. Tao H, Guo S, Ge T, Kendrick KM, Xue Z, Liu Z, et al. Depression uncouples brain hate circuit. Mol Psychiatry (2013) 18:101-11. doi:10.1038/mp. 2011.127

55. Yamagishi N, Goda N, Callan DE, Anderson SJ, Kawato M. Attentional shifts towards an expected visual target alter the level of alpha-band oscillatory activity in the human calcarine cortex. Brain Res Cogn Brain Res (2005) 25:799-809. doi:10.1016/j.cogbrainres.2005.09.006

56. Giora E, Galbiati A, Marelli S, Zucconi M, Ferini-Strambi L. Evidence of perceptive impairment in OSA patients investigated by means of a visual search task. Cortex (2017) 95:136-42. doi:10.1016/j.cortex.2017.08.004

57. Moghimi S, Ahmadraji A, Sotoodeh H, Sadeghniat K, Maghsoudipour M, Fakhraie G, et al. Retinal nerve fiber thickness is reduced in sleep apnea syndrome. Sleep Med (2013) 14:53-7. doi:10.1016/j.sleep.2012.07.004

58. Tsang CS, Chong SL, Ho CK, Li MF. Moderate to severe obstructive sleep apnoea patients is associated with a higher incidence of visual field defect. Eye (Lond) (2006) 20:38-42. doi:10.1038/sj.eye.6701785

59. Xin C, Zhang W, Wang L, Yang D, Wang J. Changes of visual field and optic nerve fiber layer in patients with OSAS. Sleep Breath (2015) 19:129-34. doi:10.1007/s11325-014-0978-9
60. Chan KC, Shi L, So HK, Wang D, Liew AW, Rasalkar DD, et al. Neurocognitive dysfunction and grey matter density deficit in children with obstructive sleep apnoea. Sleep Med (2014) 15:1055-61. doi:10.1016/j.sleep.2014.04.011

61. Tregear S, Reston J, Schoelles K, Phillips B. Obstructive sleep apnea and risk of motor vehicle crash: systematic review and meta-analysis. J Clin Sleep Med (2009) 5:573-81.

62. Tahmasian M, Rosenzweig I, Eickhoff SB, Sepehry AA, Laird AR, Fox PT, et al. Structural and functional neural adaptations in obstructive sleep apnea: an activation likelihood estimation meta-analysis. Neurosci Biobehav Rev (2016) 65:142-56. doi:10.1016/j.neubiorev.2016.03.026

63. Gupta MA, Simpson FC. Obstructive sleep apnea and psychiatric disorders: a systematic review. J Clin Sleep Med (2015) 11:165-75. doi:10.5664/jcsm.4466

64. Wang L, Li K, Zhang QE, Jin Z, Dai WJ, Su YA, et al. Interhemispheric functional connectivity and its relationships with clinical characteristics in major depressive disorder: a resting state fMRI study. PLoS One (2013) 8:e60191. doi:10.1371/journal.pone.0060191

65. Guo W, Liu F, Xue Z, Gao K, Liu Z, Xiao C, et al. Decreased interhemispheric coordination in treatment-resistant depression: a resting-state fMRI study. PLoS One (2013) 8:e71368. doi:10.1371/journal.pone.0071368

Conflict of Interest Statement: The authors declare that the research was conducted in the absence of any commercial or financial relationships that could be construed as a potential conflict of interest.

Copyright (c) 2018 Liu, Zhang, Li, Chen, Huang, Zhang, Huang, Liu and Yang. This is an open-access article distributed under the terms of the Creative Commons Attribution License (CC BY). The use, distribution or reproduction in other forums is permitted, provided the original author(s) and the copyright owner are credited and that the original publication in this journal is cited, in accordance with accepted academic practice. No use, distribution or reproduction is permitted which does not comply with these terms. 